Mathematical Sciences and Applications

E-NOTES

MATHEMATICAL

SCIENCES

AND APPLICATIONS

E-NOTES

https://doi.org/10.36753/mathenot.778766

9 (2) 81-94 (2021) - Research Article

ISSN: $2147-6268$

(C)MSAEN

\title{
Numerical Investigation of Modified Fornberg Whitham Equation
}

\author{
Nuri Murat Yağmurlu*, Ersin Yıldız, Yusuf Uçar and Alaattin Esen
}

\begin{abstract}
The aim of this study is to obtain numerical solutions of the modified Fornberg Whitham equation via collocation finite element method combined with operator splitting method. The splitting method is used to convert the original equation into two sub equations including linear and nonlinear part of the equation as a slight modification of splitting idea. After splitting progress, collocation method is used to reduce the sub equations into algebraic equation systems. For this purpose, quintic B-spline base functions are used as a polynomial approximation for the solution. The effectiveness and efficiency of the method and accuracy of the results are measured with the error norms $L_{2}$ and $L_{\infty}$. The presentations of the numerical results are shown by graphics as well.
\end{abstract}

Keywords: Modified Fornberg Whitham equation; Collocation; Quintic B-spline; Strang-splitting; Stability; FEM.

AMS Subject Classification (2020): Primary: 35Q51; Secondary: 74J35; 33F10.

*Corresponding author

\section{Introduction}

In our world, dynamic examples are based on change such as motion, electricity, heat and light, acoustics, birth and death rates, temperature, pressure and calculus is a mathematical theory concerned with this very change. All of these can be modeled appropriately using the language of mathematics. Fractional and integer orders differential equations are one of the most important and amazing parts of this language as far as science and engineering are concerned. Today, differential equations are fundamental mathematical tools representing a relationship between a continuously varying quantity and its rate of change and are used in most areas of science and technology. Thus, it is practically important for researchers to solve equations so that the behavior of the systems can be accurately studied.

After gaining their important place in our lives, ordinary and fractional differential equations have begun to get attention of many scientists. In order to share their researches, numerous articles, studies, books, reviews were published and numerous conferences, workshops, meetings were held by scientists. Meanwhile, for ordinary differential equations, new articles, new approaches and new techniques have drawn attention.

Let us consider the Fornberg-Whitham equation given by [9] 


$$
u_{t}-u_{x x t}+u_{x}+u u_{x}=3 u_{x} u_{x x}+u u_{x x x}, \quad t>0, x>0,
$$

and first proposed by Whitham in 1967 for studying the qualitative behavior of wave breaking. In 1978, Fornberg and Whitham [6] obtained a peaked solution consisting of an arbitrary constant. By modifying the nonlinear term $u u_{x}$ in Eq.(1.1) as $u^{2} u_{x}$, He et al.proposed in [9] the modified Fornberg-Whitham equation as follows

$$
u_{t}-u_{x x t}+u_{x}+u^{2} u_{x}=3 u_{x} u_{x x}+u u_{x x x}, t>0, x>0 .
$$

Here, $u(x ; t)$ is the fluid velocity, $t$ is the time and $x$ is the spatial variable.

Before starting the process, let us take a brief look at research works on the obtaining approximate and numerical solutions of Fornberg-Whitham equation; as an analytical technique Abidi and Omrani [1] have applied homotopy analysis method (HAM) and presented many comparisons with other methods. Zhou and Tian, [16] have obtained kink-like wave solutions and antikink-like wave solutions of the equation by bifurcation method. Homotopy perturbation method (HPM)[7] reduced differential transform method [10], and phase portrait analytical technology [5] have been used to obtain exact and numerical solutions of Fornberg-Whitham equation. In [9], He et al investigated modified Fornberg Whitham equation and obtained some peakons and solitary waves of the equation. In [14], Ray and Gupta have solved the modified version of the equation using a new wavelet method based on the Hermit wavelet expansion. Boutarfa et al. have applied reproducing Kernel Hilbert space method to classical, modified and fractional types of the Fornberg Whitham equation. Also Lu [11] has applied He's variational iteration method and Biazar [3] has solved Fornberg-Whitham type equations via homotopy perturbation method (HPM). Nuseir [4] has applied a unified approach for finding soliton solutions to the modified Fornberg-Whitham equation.

In this study, numerical solutions of modified Forberg Whitham equation are investigated in order to make a contribution to the literature. In the way of our aim, two effective methods, namely finite element and splitting methods whose roots are very old, are combined. The spline basis used in the finite element method are selected as quintic B-spline bases considering the highest order derivative seen in the equation. Stability analysis which is the most important part of numerical methods of the newly obtained numerical scheme, is discussed with Von Neumann stability analysis that is widely known and used. In the last part numerical examples are given. In addition to the newly obtained results, comparison tables with different studies in the literature are presented. In addition, numerical results were expressed in graphs for visual comparisons.

\section{Strang Splitting Method}

The splitting technique which we are going to use for numerical computations is the second order symmetric technique proposed by Strang [15] and sometimes known as Strang-Marchuk [12]. Strang splitting is generally used to accelerate computations for problems containing operators on very different time scales, for instance, chemical reactions in fluid dynamics, and to solve multidimensional partial differential equations. Strang splitting is the second order method so that the method provides accurate and efficient results. Strang splitting algorithm is as follows :

$$
\begin{array}{rccc}
\frac{\partial u^{*}(t)}{\partial t} & =A u^{*}(t), & t^{n} \leq t \leq t^{n+\frac{1}{2}}, & u^{*}\left(t^{n}\right)=u_{n}^{0}, \\
\frac{\partial u^{* *}(t)}{\partial t} & =B u^{* *}(t), & t^{n} \leq t \leq t^{n+1}, & u^{* *}\left(t^{n}\right)=u^{*}\left(t^{n+\frac{1}{2}}\right), \\
\frac{\partial u^{* * *}(t)}{\partial t}=A u^{* * *}(t), & t^{n+\frac{1}{2}} \leq t \leq t^{n+1}, & u^{* * *}\left(t^{n+\frac{1}{2}}\right)=u^{* *}\left(t^{n}\right),
\end{array}
$$

where $t^{n+\frac{1}{2}}=t^{n}+\frac{T}{2}$ and $u_{n}^{0}=u_{0}$, and the approximation for the next time step $t^{n+1}$ is defined as $u_{n}^{0}=u^{* * *}\left(t^{n+1}\right)$.

\section{Finite Element Quintic B-Spline Collocation Method}

For the numerical calculations, the solution domain of the problem is restricted over the interval $x_{\text {left }} \leq x \leq$ $x_{\text {right }}$. The interval is partitioned into uniformly-sized finite elements of length $h$ by the knots $x_{m}$ such that

$$
x_{\text {left }}=x_{0}<x_{1}<\cdots<x_{N}=x_{\text {right }} .
$$

The set of quintic B-spline functions 


$$
\left\{\varphi_{-2}(x), \varphi_{-1}(x), \ldots, \varphi_{N+1}(x), \varphi_{N+2}(x)\right\}
$$

forms a basis over the problem domain $\left[x_{l e f t}, x_{\text {right }}\right]$. We seek the numerical solution $u_{\text {numeric }}(x, t)$ to the exact solution $u_{\text {exact }}(x, t)$ in the form of

$$
u_{\text {numeric }}(x, t)=\sum_{m=-2}^{N+2} \delta_{m}(t) \varphi_{m}(x),
$$

where $\delta_{m}(t)$ are time dependent parameters to be determined from the boundary and collocation conditions.

Quintic B-splines $\varphi_{m}(x)$ for $(m=-2(1) N+2)$, at the knots $x_{m}$ are defined over the interval $[a, b]$ in [13] as follows

$$
\varphi_{m}(x)=\frac{1}{h^{5}} \begin{cases}\left(x-x_{m-3}\right)^{5}, & {\left[x_{m-3}, x_{m-2}\right]} \\ \left(x-x_{m-3}\right)^{5}-6\left(x-x_{m-2}\right)^{5}, & {\left[x_{m-2}, x_{m-1}\right]} \\ \left(x-x_{m-3}\right)^{5}-6\left(x-x_{m-2}\right)^{5}+15\left(x-x_{m-1}\right)^{5}, & {\left[x_{m-1}, x_{m}\right]} \\ \left(x-x_{m-3}\right)^{5}-6\left(x-x_{m-2}\right)^{5}+15\left(x-x_{m-1}\right)^{5} & \\ -20\left(x-x_{m}\right)^{5}, & {\left[x_{m}, x_{m+1}\right]} \\ \left(x-x_{m-3}\right)^{5}-6\left(x-x_{m-2}\right)^{5}+15\left(x-x_{m-1}\right)^{5} & \\ -20\left(x-x_{m}\right)^{5}+15\left(x-x_{m+1}\right)^{5}, & {\left[x_{m+1}, x_{m+2}\right]} \\ \left(x-x_{m-3}\right)^{5}-6\left(x-x_{m-2}\right)^{5}+15\left(x-x_{m-1}\right)^{5} & \\ -20\left(x-x_{m}\right)^{5}+15\left(x-x_{m+1}\right)^{5}-6\left(x-x_{m+2}\right)^{5}, & {\left[x_{m+2}, x_{m+3}\right]} \\ 0, & \text { otherwise. }\end{cases}
$$

Each quintic B-spline covers six elements so that each element $\left[x_{m}, x_{m+1}\right]$ is covered by six quintic B-splines. Substituting trial function (3.2) into Eq. (3.3), the nodal values of $u_{\text {numeric }}, u_{\text {numeric }}^{\prime}, u_{\text {numeric }}^{\prime \prime}, u_{\text {numeric }}^{\prime \prime \prime}$ at the knots $x_{m}$ are obtained in terms of the element parameters $\delta_{m}$ by

$$
\begin{aligned}
& u_{\text {numeric }}\left(x_{m}\right)=\left(u_{\text {numeric }}\right)_{m}=\delta_{m-2}+26 \delta_{m-1}+66 \delta_{m}+26 \delta_{m+1}+\delta_{m+2}, \\
& u_{\text {numeric }}^{\prime}\left(x_{m}\right)=\left(u_{\text {numeric }}\right)_{m}^{\prime}=\frac{5}{h}\left(-\delta_{m-2}-10 \delta_{m-1}+10 \delta_{m+1}+\delta_{m+2}\right), \\
& u_{\text {numeric }}^{\prime \prime}\left(x_{m}\right)=\left(u_{\text {numeric }}\right)_{m}^{\prime \prime}=\frac{20}{h^{2}}\left(\delta_{m-2}+2 \delta_{m-1}-6 \delta_{m}+2 \delta_{m+1}+\delta_{m+2}\right), \\
& u_{\text {numeric }}^{\prime \prime \prime}\left(x_{m}\right)=\left(u_{\text {numeric }}\right)_{m}^{\prime \prime \prime}=\frac{60}{h^{3}}\left(\delta_{m-2}-2 \delta_{m-1}+2 \delta_{m+1}-\delta_{m+2}\right),
\end{aligned}
$$

where the symbols '," and "' represent the first, the second and the third order differentiation with respect to $x$, respectively. The splines $\varphi_{m}(x)$ and their four principle derivatives vanish outside the interval $\left[x_{m-3}, x_{m+3}\right]$.

\subsection{Time split discretization}

The time splitting of modified Fornberg-Whitham equation is carried out as follows

$$
\begin{aligned}
& u_{t}-u_{x x t}+u_{x}=0, \\
& u_{t}-u_{x x t}+u^{2} u_{x}-3 u_{x} u_{x x}-u u_{x x x}=0,
\end{aligned}
$$

and the values of $u_{\text {numeric }}, u_{\text {numeric }}^{\prime}, u_{\text {numeric }}^{\prime \prime}$ and $u_{\text {numeric }}^{\prime \prime \prime}$ computed at collocation points $x_{m}$ are used in (3.5). After some basic operations, we obtain the following system of the first order ordinary differential equations as 
follows

$$
\begin{aligned}
& \dot{\delta}_{m-2}+ 26 \dot{\delta}_{m-1}+66 \dot{\delta}_{m}+26 \dot{\delta}_{m+1}+\dot{\delta}_{m+2}-\frac{20}{h^{2}}\left(\dot{\delta}_{m-2}+2 \dot{\delta}_{m-1}-6 \dot{\delta}_{m}+2 \dot{\delta}_{m+1}+\dot{\delta}_{m+2}\right) \\
&+\frac{5}{h}\left(-\delta_{m-2}-10 \delta_{m-1}+10 \delta_{m+1}+\delta_{m+2}\right)=0 \\
& \dot{\delta}_{m-2}+ 26 \dot{\delta}_{m-1}+66 \dot{\delta}_{m}+26 \dot{\delta}_{m+1}+\dot{\delta}_{m+2}-\frac{20}{h^{2}}\left(\dot{\delta}_{m-2}+2 \dot{\delta}_{m-1}-6 \dot{\delta}_{m}+2 \dot{\delta}_{m+1}+\dot{\delta}_{m+2}\right) \\
&+z_{m}^{2} \frac{5}{h}\left(-\delta_{m-2}-10 \delta_{m-1}+10 \delta_{m+1}+\delta_{m+2}\right)-3 g_{m} \frac{20}{h^{2}}\left(\delta_{m-2}+2 \delta_{m-1}-6 \delta_{m}+2 \delta_{m+1}+\delta_{m+2}\right) \\
&-z_{m} \frac{60}{h^{3}}\left(\delta_{m-2}-2 \delta_{m-1}+2 \delta_{m+1}-\delta_{m+2}\right)=0 .
\end{aligned}
$$

Here $z_{m}$ and $g_{m}$ are linearized terms given as follows

$$
\begin{aligned}
& z_{m}=\delta_{m-2}+26 \delta_{m-1}+66 \delta_{m}+26 \delta_{m+1}+\delta_{m+2} \\
& g_{m}=\frac{5}{h}\left(-\delta_{m-2}-10 \delta_{m-1}+10 \delta_{m+1}+\delta_{m+2}\right) .
\end{aligned}
$$

In the equation given above, if we use the Crank-Nicolson formula instead of $\delta_{m}$ and the forward difference formula instead of $\dot{\delta_{m}}$

$$
\begin{aligned}
\delta_{m} & =\frac{1}{2}\left(\delta_{m}^{n+1}+\delta_{m}^{n}\right), \\
\dot{\delta_{m}} & =\frac{\delta_{m}^{n+1}-\delta_{m}^{n}}{\Delta t},
\end{aligned}
$$

we obtain a recurrent relationship between the unknown time-level parameters $\delta_{m}^{n+1}$ and the known time-levels operators $\delta_{m}^{n}$ as follows:

$$
a_{1} \delta_{m-2}^{n+1}+a_{2} \delta_{m-1}^{n+1}+a_{3} \delta_{m}^{n+1}+a_{4} \delta_{m+1}^{n+1}+a_{5} \delta_{m+2}^{n+1}=a_{5} \delta_{m-2}^{n}+a_{4} \delta_{m-1}^{n}+a_{3} \delta_{m}^{n}+a_{2} \delta_{m+1}^{n}+a_{1} \delta_{m+2}^{n},
$$

where

$$
\begin{aligned}
& a_{1}=\frac{1}{\Delta t}-\frac{20}{\Delta t h^{2}}-\frac{5}{2 h}, \\
& a_{2}=\frac{26}{\Delta t}-\frac{40}{\Delta t h^{2}}-\frac{25}{h}, \\
& a_{3}=\frac{66}{\Delta t}-\frac{120}{\Delta t h^{2}}, \\
& a_{4}=\frac{26}{\Delta t}-\frac{40}{\Delta t h^{2}}+\frac{25}{h}, \\
& a_{5}=\frac{1}{\Delta t}-\frac{20}{\Delta t h^{2}}+\frac{5}{2 h},
\end{aligned}
$$

and

$$
b_{1} \delta_{m-2}^{n+1}+b_{2} \delta_{m-1}^{n+1}+b_{3} \delta_{m}^{n+1}+b_{4} \delta_{m+1}^{n+1}+b_{5} \delta_{m+2}^{n+1}=b_{6} \delta_{m-2}^{n}+b_{7} \delta_{m-1}^{n}+b_{8} \delta_{m}^{n}+b_{9} \delta_{m+1}^{n}+b_{10} \delta_{m+2}^{n}
$$


where

$$
\begin{array}{ll}
b_{1}=\frac{1}{\triangle t}-\frac{20}{\triangle t h^{2}}-\frac{5 z_{m}^{2}}{2 h}-\frac{30 g_{m}}{h^{2}}+\frac{30 z_{m}}{h^{3}}, & b_{6}=\frac{1}{\triangle t}-\frac{20}{\triangle t h^{2}}+\frac{5 z_{m}^{2}}{2 h}+\frac{30 g_{m}}{h^{2}}-\frac{30 z_{m}}{h^{3}}, \\
b_{2}=\frac{26}{\triangle t}-\frac{40}{\triangle t h^{2}}-\frac{25 z_{m}^{2}}{h}-\frac{60 g_{m}}{h^{2}}+\frac{60 z_{m}}{h^{3}}, & b_{7}=\frac{26}{\triangle t}-\frac{40}{\triangle t h^{2}}+\frac{25 z_{m}^{2}}{h}+\frac{60 g_{m}}{h^{2}}+\frac{60 z_{m}}{h^{3}}, \\
b_{3}=\frac{66}{\triangle t}+\frac{120}{\triangle t h^{2}}+\frac{180 g_{m}}{h^{2}}, & b_{8}=\frac{66}{\triangle t}+\frac{120}{\triangle t h^{2}}-\frac{180 g_{m}}{h^{2}}, \\
b_{4}=\frac{26}{\triangle t}-\frac{40}{\triangle t h^{2}}+\frac{25 z_{m}^{2}}{h}-\frac{60 g_{m}}{h^{2}}+\frac{60 z_{m}}{h^{3}}, & b_{9}=\frac{26}{\triangle t}-\frac{40}{\triangle t h^{2}}-\frac{25 z_{m}^{2}}{h}+\frac{60 g_{m}}{h^{2}}-\frac{60 z_{m}}{h^{3}}, \\
b_{5}=\frac{1}{\triangle t}-\frac{20}{\triangle t h^{2}}+\frac{5 z_{m}^{2}}{2 h}-\frac{30 g_{m}}{h^{2}}-\frac{30 z_{m}}{h^{3}}, & b_{10}=\frac{1}{\triangle t}-\frac{20}{\triangle t h^{2}}-\frac{5 z_{m}^{2}}{2 h}+\frac{30 g_{m}}{h^{2}}+\frac{30 z_{m}}{h^{3}},
\end{array}
$$

In the equation system given by Eq. (3.2), the number of unknown variables is $(N+5)$, and number the of equations is $(N+1)$; so, to obtain a solvable system, we should eliminate $\delta_{-2}, \delta_{-1}, \delta_{N+1}$, and $\delta_{N+2}$ from the systems using the values of $\left(u_{\text {numeric }}\right)_{m}$ and $\left(u_{\text {numeric }}\right)_{m}^{\prime}$ at the boundary points of the interval. In the end of this elimination operation, we can obtain an equation system of the type $(N+1) \times(N+1)$, and then Strang method is applied to obtain schemes. In order to solve these systems, we need the initial vector $\delta_{m}^{0}$

\subsection{Initial state}

In the previous section, we have constructed an iterative equation system. To begin this iteration, we start with the initial vector. In this section, we are going to obtain this vector. Let us call the initial vector $\left(u_{\text {numeric }}\right)_{0}=$ $\left(\delta_{0}, \delta_{1}, \ldots \delta_{N-1}, \delta_{N}\right)^{T}$, which is derived from the initial conditions of the problem, and the approximate solution for $\left(u_{\text {numeric }}\right)_{0},\left(u_{\text {numeric }}\right)_{N}$ as follows:

$$
\left(u_{\text {numeric }}\right)_{N}(x, 0)=\left(u_{\text {exact }}\right)(x, 0)=\sum_{m=-2}^{N+2} \delta_{m}(0) \varphi_{m}(x) .
$$

Thus, if this system is rewritten clearly, a penta-diagonal matrices emerges of the following form:

$$
\left[\begin{array}{cccccccc}
54 & 60 & 6 & & & & & \\
25.25 & 67.50 & 26.25 & 1 & & & & \\
1 & 26 & 66 & 26 & 1 & & & \\
& 1 & 26 & 66 & 26 & 1 & & \\
& & & & \ddots & & & \\
& & & 1 & 26 & 66 & 26 & 1 \\
& & & & 1 & 26.25 & 67.50 & 25.25 \\
& & & & & 6 & 60 & 54
\end{array}\right]\left[\begin{array}{c}
\delta_{0} \\
\delta_{1} \\
\delta_{2} \\
\\
\vdots \\
\\
\delta_{N-1} \\
\delta_{N}
\end{array}\right]=\left[\begin{array}{c}
U_{0} \\
U_{1} \\
U_{2} \\
\\
\vdots \\
\\
U_{N-1} \\
U_{N}
\end{array}\right]
$$

So, the required initial vector to solve Equation (3.2) has been derived.

\section{Stability Analysis}

This section of the presented work is concerned with stability analysis of numerical scheme given in Eq. (1.2). For this purpose, we will use Von Neumann stability analysis, the importance of the analysis is that, it provides a way to extract information about the eigenvalue without knowing exact eigenvalues themselves. Let us recall 
obtained numerical scheme in a more explicit expression using the following table;

\begin{tabular}{|c|c|}
\hline The first system (A) & $\delta_{m-2}^{n+1}\left[\frac{1}{\Delta t}-\frac{20}{\Delta t h^{2}}-\frac{5}{2 h}\right]$ \\
\hline$\delta_{m-2}^{n+1}\left[\frac{1}{\Delta t}-\frac{20}{\Delta t h^{2}}-\frac{5}{2 h}\right]$ & $\delta_{m-2}^{n}\left[\frac{1}{\Delta t}-\frac{20}{\Delta t h^{2}}+\frac{5}{2 h}\right]$ \\
\hline$\delta_{m-1}^{n+1}\left[\frac{26}{\Delta t}-\frac{40}{\Delta t h^{2}}-\frac{25}{2 h}\right]$ & $\delta_{m-1}^{n}\left[\frac{26}{\Delta t}-\frac{40}{\Delta t h^{2}}+\frac{25}{2 h}\right]$ \\
\hline$\delta_{m}^{n+1}\left[\frac{66}{\Delta t}-\frac{120}{\Delta t h^{2}}\right]$ & $\delta_{m}^{n}\left[\frac{66}{\Delta t}-\frac{120}{\Delta t h^{2}}\right]$ \\
\hline$\delta_{m+1}^{n+1}\left[\frac{26}{\Delta t}-\frac{40}{\Delta t h^{2}}+\frac{25}{2 h}\right]$ & $\delta_{m+1}^{n}\left[\frac{26}{\Delta t}-\frac{40}{\Delta t h^{2}}-\frac{25}{2 h}\right]$ \\
\hline$\delta_{m-2}^{n+1}\left[\frac{1}{\Delta t}-\frac{20}{\Delta t h^{2}}+\frac{5}{2 h}\right]$ & $\delta_{m-2}^{n}\left[\frac{1}{\Delta t}-\frac{20}{\Delta t h^{2}}-\frac{5}{2 h}\right]$ \\
\hline The second system (B) & $\delta_{m-2}^{n+1}\left[\frac{1}{\Delta t}-\frac{20}{\Delta t h^{2}}-\frac{5}{2 h} z_{m}^{2}-\frac{30}{h^{2}} g_{m}+\frac{30}{h^{3}} z_{m}\right]$ \\
\hline$\delta_{m-2}^{n+1}\left[\frac{1}{\Delta t}-\frac{20}{\Delta t h^{2}}-\frac{5}{2 h} z_{m}^{2}-\frac{30}{h^{2}} g_{m}+\frac{30}{h^{3}} z_{m}\right]$ & $\delta_{m-2}^{n}\left[\frac{1}{\Delta t}-\frac{20}{\Delta t h^{2}}+\frac{5}{2 h} z_{m}^{2}+\frac{30}{h^{2}} g_{m}-\frac{30}{h^{3}} z_{m}\right]$ \\
\hline$\delta_{m-1}^{n+1}\left[\frac{26}{\Delta t}-\frac{40}{\Delta t h^{2}}-\frac{25}{2 h} z_{m}^{2}-\frac{60}{h^{2}} g_{m}+\frac{60}{h^{3}} z_{m}\right]$ & $\delta_{m-1}^{n}\left[\frac{26}{\Delta t}-\frac{40}{\Delta t h^{2}}+\frac{25}{2 h} z_{m}^{2}+\frac{60}{h^{2}} g_{m}+\frac{60}{h^{3}} z_{m}\right]$ \\
\hline$\delta_{m}^{n+1}\left[\frac{66}{\Delta t}+\frac{120}{\Delta t h^{2}}+\frac{180}{h^{2}} g_{m}\right]$ & $\delta_{m}^{n}\left[\frac{66}{\Delta t}+\frac{120}{\Delta t h^{2}}-\frac{180}{h^{2}} g_{m}\right]$ \\
\hline$\delta_{m+1}^{n+1}\left[\frac{26}{\Delta t}-\frac{40}{\Delta t h^{2}}+\frac{25}{2 h} z_{m}^{2}-\frac{60}{h^{2}} g_{m}-\frac{60}{h^{3}} z_{m}\right]$ & $\delta_{m+1}^{n}\left[\frac{26}{\Delta t}-\frac{40}{\Delta t h^{2}}-\frac{25}{2 h} z_{m}^{2}+\frac{60}{h^{2}} g_{m}-\frac{60}{h^{3}} z_{m}\right]$ \\
\hline$\delta_{m-2}^{n+1}\left[\frac{1}{\Delta t}-\frac{20}{\Delta t h^{2}}+\frac{5}{2 h} z_{m}^{2}-\frac{30}{h^{2}} g_{m}-\frac{30}{h^{3}} z_{m}\right]$ & $\delta_{m-2}^{n}\left[\frac{1}{\Delta t}-\frac{20}{\Delta t h^{2}}-\frac{5}{2 h} z_{m}^{2}+\frac{30}{h^{2}} g_{m}+\frac{30}{h^{3}} z_{m}\right]$ \\
\hline
\end{tabular}

It is shown that, the first equation is more symetric than the second one. According to Von Neumann stability analysis, numerical scheme admits a solution of the form

$$
\delta_{m}^{n}=\xi^{n} e^{i w m},
$$

where $w$ is the wave number and $i=\sqrt{-1}$ and $\xi$ is an amplification factor. Before diving into the stability analysis, let us remind some useful identities

$$
\begin{aligned}
& \cos x=\frac{e^{i x}+e^{-i x}}{2}, \\
& \sin x=\frac{e^{i x}-e^{-i x}}{2 i} .
\end{aligned}
$$


Now, when we replace each solution term $\delta_{m}^{n}$ with the equation given in (4.1), we get,

The first system(A)

$$
\begin{aligned}
& \xi_{A}^{n+1} e^{i w m}\left\{\left(\frac{1}{\Delta t}-\frac{20}{\Delta t h^{2}}-\frac{5}{2 h}\right) e^{-2 i w}+\left(\frac{1}{\Delta t}-\frac{20}{\Delta t h^{2}}+\frac{5}{2 h}\right) e^{2 i w}+\left(\frac{66}{\Delta t}-\frac{120}{\Delta t h^{2}}\right)\right. \\
& \left.+\left(\frac{26}{\Delta t}-\frac{40}{\Delta t h^{2}}-\frac{25}{2 h}\right) e^{-i w}+\left(\frac{26}{\Delta t}-\frac{40}{\Delta t h^{2}}+\frac{25}{2 h}\right) e^{i w}\right\}=\xi_{A}^{n} e^{i w m}\left\{\left(\frac{1}{\Delta t}-\frac{20}{\Delta t h^{2}}+\frac{5}{2 h}\right) e^{-2 i w}\right. \\
& \left.+\left(\frac{1}{\Delta t}-\frac{20}{\Delta t h^{2}}-\frac{5}{2 h}\right) e^{2 i w}+\left(\frac{66}{\Delta t}-\frac{120}{\Delta t h^{2}}\right)+\left(\frac{26}{\Delta t}-\frac{40}{\Delta t h^{2}}+\frac{25}{2 h}\right) e^{-i w}+\left(\frac{26}{\Delta t}-\frac{40}{\Delta t h^{2}}-\frac{25}{2 h}\right) e^{i w}\right\},
\end{aligned}
$$

The second system(B)

$$
\begin{aligned}
& \xi_{B}^{n+1} e^{i w m}\left\{\left(\frac{1}{\Delta t}-\frac{20}{\Delta t h^{2}}-\frac{5}{2 h} z_{m}^{2}-\frac{30}{h^{2}} g_{m}+\frac{30}{h^{3}} z_{m}\right) e^{-2 i w}+\left(\frac{1}{\Delta t}-\frac{20}{\Delta t h^{2}}+\frac{5}{2 h} z_{m}^{2}-\frac{30}{h^{2}} g_{m}-\frac{30}{h^{3}} z_{m}\right) e^{2 i w}\right. \\
& +\left(\frac{66}{\Delta t}+\frac{120}{\Delta t h^{2}}+\frac{180}{h^{2}} g_{m}\right)+\left(\frac{26}{\Delta t}-\frac{40}{\Delta t h^{2}}-\frac{25}{2 h} z_{m}^{2}-\frac{60}{h^{2}} g_{m}+\frac{60}{h^{3}} z_{m}\right) e^{-i w} \\
& \left.+\left(\frac{26}{\Delta t}-\frac{40}{\Delta t h^{2}}+\frac{25}{2 h} z_{m}^{2}-\frac{60}{h^{2}} g_{m}-\frac{60}{h^{3}} z_{m}\right) e^{i w}\right\}=\xi_{B}^{n} e^{i w m}\left\{\left(\frac{1}{\Delta t}-\frac{20}{\Delta t h^{2}}+\frac{5}{2 h} z_{m}^{2}+\frac{30}{h^{2}} g_{m}-\frac{30}{h^{3}} z_{m}\right) e^{-2 i w}\right. \\
& +\left(\frac{1}{\Delta t}-\frac{20}{\Delta t h^{2}}-\frac{5}{2 h} z_{m}^{2}+\frac{30}{h^{2}} g_{m}+\frac{30}{h^{3}} z_{m}\right) e^{2 i w}+\left(\frac{66}{\Delta t}+\frac{120}{\Delta t h^{2}}-\frac{180}{h^{2}} g_{m}\right) \\
& \left.+\left(\frac{26}{\Delta t}-\frac{40}{\Delta t h^{2}}+\frac{25}{2 h} z_{m}^{2}+\frac{60}{h^{2}} g_{m}+\frac{60}{h^{3}} z_{m}\right) e^{-i w}+\left(\frac{26}{\Delta t}-\frac{40}{\Delta t h^{2}}-\frac{25}{2 h} z_{m}^{2}+\frac{60}{h^{2}} g_{m}-\frac{60}{h^{3}} z_{m}\right) e^{i w}\right\}
\end{aligned}
$$

and after some calculations, we get

The first system(A)

$$
\begin{aligned}
& \xi_{A}\left\{\frac{66}{\Delta t}-\frac{120}{\Delta t h^{2}}+\left(\frac{1}{\Delta t}-\frac{20}{\Delta t h^{2}}\right) \cos (2 w)+\left(\frac{26}{\Delta t}-\frac{40}{\Delta t h^{2}}\right) \cos (w)+\right. \\
& \left.i\left(\frac{5}{2 h} \sin (2 w)+\frac{25}{2 h} \sin (w)\right)\right\}=\frac{66}{\Delta t}-\frac{120}{\Delta t h^{2}}+\left(\frac{1}{\Delta t}-\frac{20}{\Delta t h^{2}}\right) \cos (2 w) \\
& +\left(\frac{26}{\Delta t}-\frac{40}{\Delta t h^{2}}\right) \cos (w)-i\left(\frac{5}{2 h} \sin (2 w)+\frac{25}{2 h} \sin (w)\right),
\end{aligned}
$$

The second system(B)

$$
\begin{aligned}
& \xi_{B}\left\{\frac{33}{\Delta t}+\frac{60}{\Delta t h^{2}}+\frac{90}{h^{2}} g_{m}+\left(\frac{1}{\Delta t}-\frac{20}{\Delta t h^{2}}-\frac{30}{h^{2}} g_{m}\right) \cos (2 w)+\left(\frac{26}{\Delta t}-\frac{40}{\Delta t h^{2}}-\frac{60}{h^{2}} g_{m}\right) \cos (w)\right. \\
& \left.+i\left(\left(\frac{5}{2 h} z_{m}^{2}-\frac{30}{h^{3}} z_{m}\right) \sin (2 w)+\left(\frac{25}{2 h} z_{m}^{2}-\frac{60}{h^{3}} z_{m}\right) \sin (w)\right)\right\}= \\
& \left\{\frac{33}{\Delta t}+\frac{60}{\Delta t h^{2}}-\frac{90}{h^{2}} g_{m}+\left(\frac{1}{\Delta t}-\frac{20}{\Delta t h^{2}}+\frac{30}{h^{2}} g_{m}\right) \cos (2 w)+\left(\frac{26}{\Delta t}-\frac{40}{\Delta t h^{2}}+\frac{60}{h^{2}} g_{m}\right) \cos (w)\right. \\
& \left.-i\left(\left(\frac{5}{2 h} z_{m}^{2}-\frac{30}{h^{3}} z_{m}\right) \sin (2 w)+\left(\frac{25}{2 h} z_{m}^{2}+\frac{60}{h^{3}} z_{m}\right) \sin (w)\right)\right\}
\end{aligned}
$$


where

$$
\begin{aligned}
& p_{1}=\frac{66}{\Delta t}-\frac{120}{\Delta t h^{2}}+\left(\frac{1}{\Delta t}-\frac{20}{\Delta t h^{2}}\right) \cos (2 w)+\left(\frac{26}{\Delta t}-\frac{40}{\Delta t h^{2}}\right) \cos (w) \\
& p_{2}=\left(\frac{5}{2 h} \sin (2 w)+\frac{25}{2 h} \sin (w)\right)
\end{aligned}
$$

from the first equation it is obvious that

$$
\xi_{A}=\frac{p_{1}-i p_{2}}{p_{1}+i p_{2}}
$$

and

$$
\left|\xi_{A}\right| \leq 1
$$

The second one;

$$
\begin{aligned}
& q_{1}=\frac{33}{\Delta t}+\frac{60}{\Delta t h^{2}}+\frac{90}{h^{2}} g_{m}+\left(\frac{1}{\Delta t}-\frac{20}{\Delta t h^{2}}-\frac{30}{h^{2}} g_{m}\right) \cos (2 w)+\left(\frac{26}{\Delta t}-\frac{40}{\Delta t h^{2}}-\frac{60}{h^{2}} g_{m}\right) \cos (w), \\
& q_{2}=\left(\frac{5}{2 h} z_{m}^{2}-\frac{30}{h^{3}} z_{m}\right) \sin (2 w)+\left(\frac{25}{2 h} z_{m}^{2}-\frac{60}{h^{3}} z_{m}\right) \sin (w), \\
& q_{3}=\frac{33}{\Delta t}+\frac{60}{\Delta t h^{2}}-\frac{90}{h^{2}} g_{m}+\left(\frac{1}{\Delta t}-\frac{20}{\Delta t h^{2}}+\frac{30}{h^{2}} g_{m}\right) \cos (2 w)+\left(\frac{26}{\Delta t}-\frac{40}{\Delta t h^{2}}+\frac{60}{h^{2}} g_{m}\right) \cos (w), \\
& q_{4}=\left(\frac{5}{2 h} z_{m}^{2}-\frac{30}{h^{3}} z_{m}\right) \sin (2 w)+\left(\frac{25}{2 h} z_{m}^{2}+\frac{60}{h^{3}} z_{m}\right) \sin (w),
\end{aligned}
$$

and we can define it as

$$
\xi_{B}=\frac{q_{1}+i q_{2}}{q_{3}+i q_{4}}
$$

After some simplifications, we obtain the same expressions for $q_{1}^{2}+q_{2}^{2}$ and $q_{3}^{2}+q_{4}^{2}$ in the following form;

$$
\begin{aligned}
& \left(4 h^{2}\left(-30 \Delta \mathbf{t} g_{m}(2 \cos (w)+\cos (2 w)-6)+\left(26 h^{2}-40\right) \cos (w)+\left(h^{2}-20\right) \cos (2 w)+66 h^{2}+120\right)^{2}\right. \\
& \left.+25 \Delta \mathbf{t}^{2} z_{m}^{2} \sin ^{2}(w)\left(h^{2} z_{m}(2 \cos (w)+5)-24(\cos (w)+1)\right)^{2}\right) / \\
& \left(4 h^{2}\left(30 \Delta \mathbf{t} g_{m}(2 \cos (w)+\cos (2 w)-6)+\left(26 h^{2}-40\right) \cos (w)+\left(h^{2}-20\right) \cos (2 w)+66 h^{2}+120\right)^{2}\right. \\
& \left.+25 \Delta \mathbf{t}^{2} z_{m}^{2} \sin ^{2}(w)\left(h^{2} z_{m}(2 \cos (w)+5)+24(\cos (w)+1)\right)^{2}\right) .
\end{aligned}
$$

It is obivious that

$$
\left|\xi_{B}\right| \leq 1
$$

Therefore,

$$
|\xi| \leq\left|\xi_{A}^{n+1 / 2}\right|\left|\xi_{B}^{n}\right|\left|\xi_{A}^{n+1 / 2}\right| \leq 1,
$$

numerical scheme of Modified Forberg Whitham equation is unconditionally stable. 


\section{Numerical Experiments}

In this section numerical experiments of modified Fornberg-Whitham equation are presented. Firstly, the interval of the problem is chosen as $\left[x_{\text {left }}, x_{\text {right }}\right]=[-70,70]$ and the final time of the processing is chosen as $t_{\text {final }}=1$ considering the studies in the literature. Let us consider the modified Fornberg-Whitham equation

$$
u_{t}-u_{x x t}+u_{x}+u^{2} u_{x}=3 u_{x} u_{x x}+u u_{x x x}, t>0, x>0
$$

with the initial and boundary conditions

$$
\begin{aligned}
& u(x, 0)=f(x)=\frac{3}{4}(\sqrt{15}-5) \operatorname{sech}^{2}\left(\frac{1}{20} \sqrt{10(5-\sqrt{15})} x\right) . \\
& u\left(x_{\text {left }}, t\right)=g_{1}(t), u\left(x_{\text {right }}, t\right)=g_{2}(t)
\end{aligned}
$$

The exact solution of Eq.(1.2) is given by [9]

$$
u(x, t)=\frac{3}{4}(\sqrt{15}-5) \operatorname{sech}^{2}\left[\frac{1}{20} \sqrt{10(5-\sqrt{15})}(x-(5-\sqrt{15}) t)\right] .
$$

In order to test our method we have used the error norms, widely used in the literature, namely $L_{2}$ and $L_{\infty}$

$$
\begin{aligned}
& L_{\infty}=\left\|u_{\text {exact }}-u_{\text {numeric }}\right\|_{\infty}=\max \left|\left(u_{\text {exact }}\right)_{m}-\left(u_{\text {numeric }}\right)_{m}\right|, \\
& L_{2}=\left\|u_{\text {exact }}-u_{\text {numeric }}\right\|_{2}=\sqrt{N h \sum_{m=0}^{N}\left(\left|\left(u_{\text {exact }}\right)_{m}-\left(u_{\text {numeric }}\right)_{m}\right|\right)^{2}} .
\end{aligned}
$$

We first present our tables and calculate the error norms in Tables 1 and 2 by discretizing the spatial dimensions and the time domain into several segments, this will give us an idea on numerical behaviour of solutions according to the number of space and time steps and measure the accuracy. The numerical solution shows the characteristics of the problem as illustrated in Figure 1. It can be seen from the tables that as contrary to expectations of the properties collocation method, the errors have a slight increase by increasing the number of partition, increasing begins after 9 th digits. When we direct our attention to time steps, we can say that increasing number of time partition has positive effects on numerical results.

Table 1. The error norms $L_{2}$ and $L_{\infty}$ for different values of $h$ and at various times for $\Delta t=0.01$

\begin{tabular}{cllll}
\hline $\mathrm{t}$ & $\mathrm{h}=0.1$ & \multicolumn{3}{l}{$\mathrm{h}=0.05$} \\
\hline \hline & $L_{2} \times 10^{8}$ & $L_{\infty} \times 10^{8}$ & $L_{2} \times 10^{8}$ & $L_{\infty} \times 10^{8}$ \\
0.1 & 0.55596195 & 0.21513131 & 0.5611164946 & 0.2165036816 \\
0.2 & 1.11177145 & 0.43023812 & 1.1221487463 & 0.4329973602 \\
0.3 & 1.66754696 & 0.64512058 & 1.6831388218 & 0.6492883120 \\
0.4 & 2.22323537 & 0.85955243 & 2.2440439308 & 0.8651545480 \\
0.5 & 2.77880288 & 1.07344476 & 2.8048323184 & 1.0805117356 \\
0.6 & 3.33421992 & 1.28687775 & 3.3654789299 & 1.2951254291 \\
0.7 & 3.88945852 & 1.49929724 & 3.9259561214 & 1.5086576766 \\
0.8 & 4.44449195 & 1.71041000 & 4.4862449398 & 1.7210618375 \\
0.9 & 4.99929360 & 1.91990667 & 5.0463136992 & 1.9320877231 \\
1 & 5.55384043 & 2.12747406 & 5.6061474738 & 2.1417522755 \\
\hline
\end{tabular}


Table 2. The error norms $L_{2}$ and $L_{\infty}$ for different values of $h$ and at various times for $\Delta t=0.001$

\begin{tabular}{lllll}
\hline $\mathrm{t}$ & $\mathrm{h}=0.1$ & \multicolumn{3}{l}{$\mathrm{h}=0.05$} \\
\hline \hline & $L_{2} \times 10^{10}$ & $L_{\infty} \times 10^{10}$ & $L_{2} \times 10^{10}$ & $L_{\infty} \times 10^{10}$ \\
0.1 & 1.08863387 & 2.18611128 & 0.88634683 & 2.18611128 \\
0.2 & 1.43878123 & 2.27040776 & 1.34502978 & 2.27040776 \\
0.3 & 1.88191516 & 2.35795472 & 1.87740591 & 2.35795472 \\
0.4 & 2.36489451 & 2.44887749 & 2.43572593 & 2.44887749 \\
0.5 & 2.86625819 & 2.54330625 & 3.00757330 & 2.54330625 \\
0.6 & 3.37605384 & 2.64137618 & 3.58536542 & 2.64137618 \\
0.7 & 3.88927927 & 2.74322768 & 4.16866020 & 2.74322768 \\
0.8 & 4.40313619 & 2.84900659 & 4.75419250 & 2.84900659 \\
0.9 & 4.91550802 & 2.95886432 & 5.34114795 & 2.95886432 \\
1 & 5.42567395 & 3.07295818 & 5.93169442 & 3.07295818 \\
\hline
\end{tabular}

We secondly present some comparison tables between newly obtained numerical results with different papers in the literature. The comparisons also aim to reveal if the newly obtained numerical solutions create a difference according to results in the papers written on modified Fornberg Whitham equation. Table 3 presents a comparison of absolute errors between present method and Ref. [3]. For collocation method, we chose step size $h=0.01$ and $\Delta t=0.001$. and we will compare absolute errors of the presented method and with those in Refs. [2, 11]. It can be seen clearly from the Tables 4 and 5 that collocation method which is combined with splitting methods is better, faster and more reliable than the other methods.

Table 3. A comparison of the absolute norms with Ref.[3] for $h=0.01$ and $\Delta t=0.001$.

\begin{tabular}{llllll}
\hline & & $\left|u_{\text {numerical }}-u_{\text {exact }}\right|$ & $\left|u_{\text {numerical }}-u_{\text {exact }}\right|$ & Numerical & Exact \\
\hline \hline $\mathrm{x}$ & $\mathrm{t}$ & {$[3]$} & Present Method & & \\
\hline 1 & 0.03 & $2.015219 \times 10^{-4}$ & $4.61720661704 \times 10^{-11}$ & -0.82341419067 & -0.82341419072 \\
1 & 0.1 & $1.803436 \times 10^{-4}$ & $1.554346651389 \times 10^{-10}$ & -0.82678632533 & -0.82678632548 \\
2 & 0.04 & $1.626634 \times 10^{-4}$ & $6.13648021286 \times 10^{-11}$ & -0.76040231746 & -0.76040231752 \\
3 & 0.05 & $1.0812872 \times 10^{-3}$ & $7.22619741822 \times 10^{-11}$ & -0.66836680691 & -0.66836680698 \\
5 & 0.02 & $1.949816 \times 10^{-4}$ & $2.06346051357 \times 10^{-11}$ & -0.45047800491 & -0.45047800493 \\
5 & 0.08 & $1.01287753 \times 10^{-2}$ & $8.50673975705 \times 10^{-11}$ & -0.45748975536 & -0.45748975544 \\
6 & 0.06 & $9.963555 \times 10^{-4}$ & $4.87022089324 \times 10^{-11}$ & -0.35727422165 & -0.35727422170 \\
& & & & & \\
-1 & 0.03 & $2.163505 \times 10^{-4}$ & $4.25101065460 \times 10^{-11}$ & -0.82031160315 & -0.82031160319 \\
-1 & 0.1 & $1.1661657 \times 10^{-3}$ & $1.412389094568 \times 10^{-10}$ & -0.81644867016 & -0.81644867030 \\
-2 & 0.04 & $3.30110 \times 10^{-5}$ & $4.94783103377 \times 10^{-11}$ & -0.75298986512 & -0.75298986517 \\
-3 & 0.05 & $4.186843 \times 10^{-4}$ & $5.08697528545 \times 10^{-11}$ & -0.65671316425 & -0.65671316430 \\
-5 & 0.02 & $2.222605 \times 10^{-4}$ & $1.38800082539 \times 10^{-11}$ & -0.44582923727 & -0.44582923728 \\
-5 & 0.08 & $3.8572370 \times 10^{-3}$ & $5.42853539898 \times 10^{-11}$ & -0.43889625694 & -0.43889625699 \\
-6 & 0.06 & $1.2543867 \times 10^{-3}$ & $3.46204731549 \times 10^{-11}$ & -0.34508506110 & -0.34508506113 \\
\hline
\end{tabular}


Table 4. A comparison of the present values with those of Ref.[2] for $h=0.01$ and $\Delta t=0.001$.

\begin{tabular}{ccccccc}
\hline \multicolumn{5}{c}{$\mathrm{t}=0.02$} & & \multicolumn{3}{c}{$\mathrm{t}=0.04$} \\
\hline $\mathrm{x}$ & {$[2]$} & Present method & exact & {$[2]$} & Present method & exact \\
\hline \hline 2.5 & -0.7144249843 & -0.71442495966 & -0.71442495969 & -0.7165464263 & -0.71654637707 & -0.71654637713 \\
5 & -0.4504779212 & -0.61197020467 & -0.61197020470 & -0.4528101955 & -0.45281018904 & -0.45281018908 \\
7.5 & -0.2349678714 & -0.23496785320 & -0.23496785321 & -0.2364825099 & -0.23648258095 & -0.23648258097 \\
10 & -0.1107643868 & -0.11076431868 & -0.11076431869 & -0.1115481917 & -0.11154818385 & -0.11154818386 \\
& & & & & & \\
& & $\mathrm{t}=0.06$ & & & & \\
2.5 & -0.7186566715 & -0.71865665169 & -0.71865665178 & -0.7207556337 & -0.72075559660 & -0.72075559672 \\
5 & -0.4551474661 & -0.45514746646 & -0.45514746652 & -0.4574897077 & -0.45748975536 & -0.45748975544 \\
7.5 & -0.2380051612 & -0.23800516703 & -0.23800516706 & -0.2395356954 & -0.23953562521 & -0.23953562525 \\
10 & -0.1123371196 & -0.11233717189 & -0.11233717190 & -0.1131312117 & -0.11313131003 & -0.11313131005 \\
& & & & & & \\
\hline 2.5 & -0.7228430209 & -0.72284302499 & -0.72284302514 & & & \\
5 & -0.4598370439 & -0.45983697264 & -0.45983697274 & & & \\
7.5 & -0.2410739190 & -0.24107396885 & -0.24107396890 & & & \\
10 & -0.1139306377 & -0.11393062556 & -0.11393062558 & & & \\
\hline
\end{tabular}

Table 5. A comparison of the absolute norms for $h=0.01$ and $\Delta t=0.001$.

\begin{tabular}{llllll}
\hline $\mathrm{t}$ & & $\mathrm{x}=2.5$ & $\mathrm{x}=5$ & $\mathrm{x}=7.5$ & $\mathrm{x}=10$ \\
\hline \hline & & & & & \\
& {$[2]$} & $2.45 \mathrm{e}-8$ & $8.36 \mathrm{e}-8$ & $1.86 \mathrm{e}-8$ & $6.82 \mathrm{e}-8$ \\
0.02 & {$[11]$} & $1.180 \mathrm{e}-4$ & $2.124 \mathrm{e}-5$ & $2.805 \mathrm{e}-5$ & $5.528 \mathrm{e}-6$ \\
& Present & $2.96266344790 \mathrm{e}-11$ & $2.06346051357 \mathrm{e}-11$ & $9.8586139252 \mathrm{e}-12$ & $3.8842817851 \mathrm{e}-12$ \\
& & & & & \\
& {$[2]$} & $4.90 \mathrm{E}-8$ & $6.4 \mathrm{e}-9$ & $7.09 \mathrm{e}-8$ & $7.9 \mathrm{e}-9$ \\
0.04 & {$[11]$} & $2.363 \mathrm{e}-4$ & $4.797 \mathrm{e}-5$ & $5.772 \mathrm{e}-5$ & $1.084 \mathrm{e}-5$ \\
& Present & $5.96940274988 \mathrm{e}-11$ & $4.18736711971 \mathrm{e}-11$ & $2.00255367844 \mathrm{e}-11$ & $7.9601325531 \mathrm{e}-12$ \\
& & & & & \\
& {$[2]$} & $1.96 \mathrm{e}-8$ & $4 . e-10$ & $6.0 \mathrm{e}-9$ & $5.21 \mathrm{e}-8$ \\
0.06 & {$[11]$} & $3.547 \mathrm{e}-4$ & $8.029 \mathrm{e}-5$ & $8.902 \mathrm{e}-5$ & $1.591 \mathrm{e}-5$ \\
& Present & $9.00313157359 \mathrm{e}-11$ & $6.33545993445 \mathrm{e}-11$ & $3.01760283428 \mathrm{e}-11$ & $1.20318338626 \mathrm{e}-11$ \\
& & & & & \\
& {$[2]$} & $3.70 \mathrm{e}-8$ & $4.76 \mathrm{e}-8$ & $7.06 \mathrm{e}-8$ & $9.83 \mathrm{e}-8$ \\
0.08 & {$[11]$} & $4.731 \mathrm{e}-4$ & $1.183 \mathrm{e}-4$ & $1.220 \mathrm{e}-4$ & $2.071 \mathrm{e}-5$ \\
& Present & $1.205959776485 \mathrm{e}-10$ & $8.50673975705 \mathrm{e}-11$ & $4.08358624693 \mathrm{e}-11$ & $1.62783536650 \mathrm{e}-11$ \\
& & & & & \\
& {$[2]$} & $4.2 \mathrm{e}-9$ & $7.11 \mathrm{e}-8$ & $4.98 \mathrm{e}-8$ & $1.23 \mathrm{e}-8$ \\
0.1 & {$[11]$} & $5.914 \mathrm{e}-4$ & $1.622 \mathrm{e}-4$ & $1.565 \mathrm{e}-4$ & $2.524 \mathrm{e}-5$ \\
& Present & $1.511807345977 \mathrm{e}-10$ & $1.072741895314 \mathrm{e}-10$ & $5.14761289150 \mathrm{e}-11$ & $2.05421096799 \mathrm{e}-11$ \\
\hline
\end{tabular}

Lastly, we present some simulations for numerical results. To do that, we begin our simulation with choosing $h=0.1, \Delta t=0.01, t_{\text {final }}=10$ and illustrate in Figure 2 for different times simultaneously. Also, we present a table 
including the position of pick point of the wave according to time for $h=0.1, \Delta t=0.01, t_{\text {final }}=10$. in Table 6 . It can be seen from table and figures that the wave moves to the right side of axis slowly and keeps its shape.
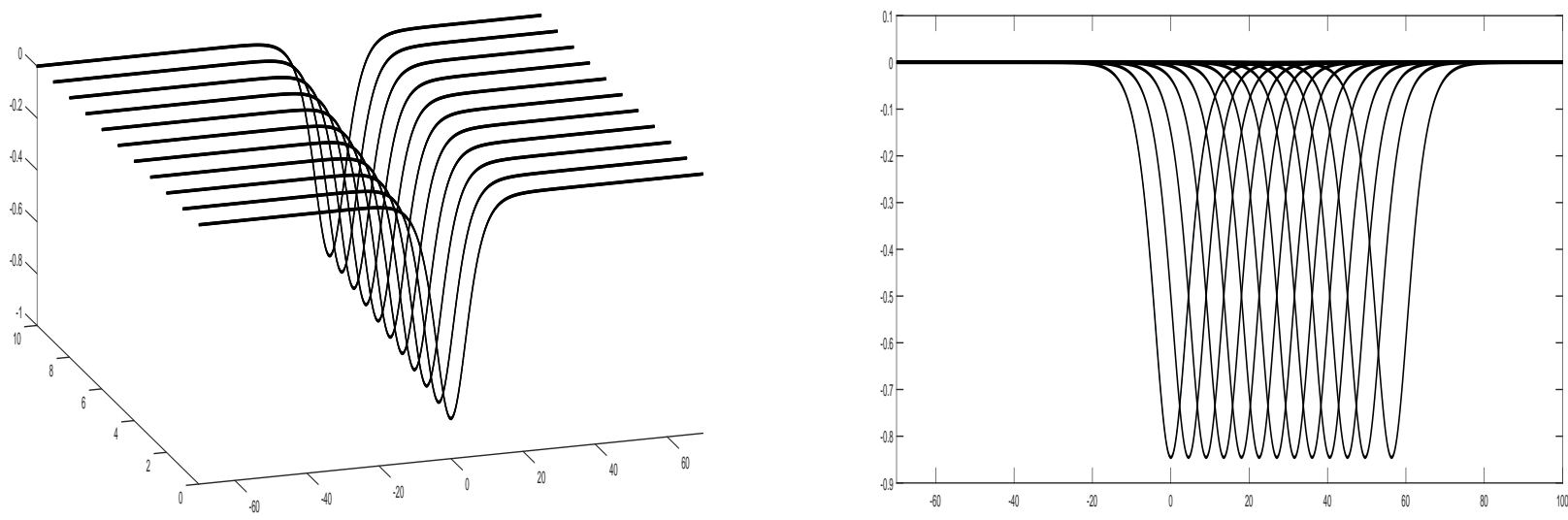

Figure 1. The graphics of numerical solutions of Modified Fornberg equation for times $t=10$ and $t=50$, respectively.

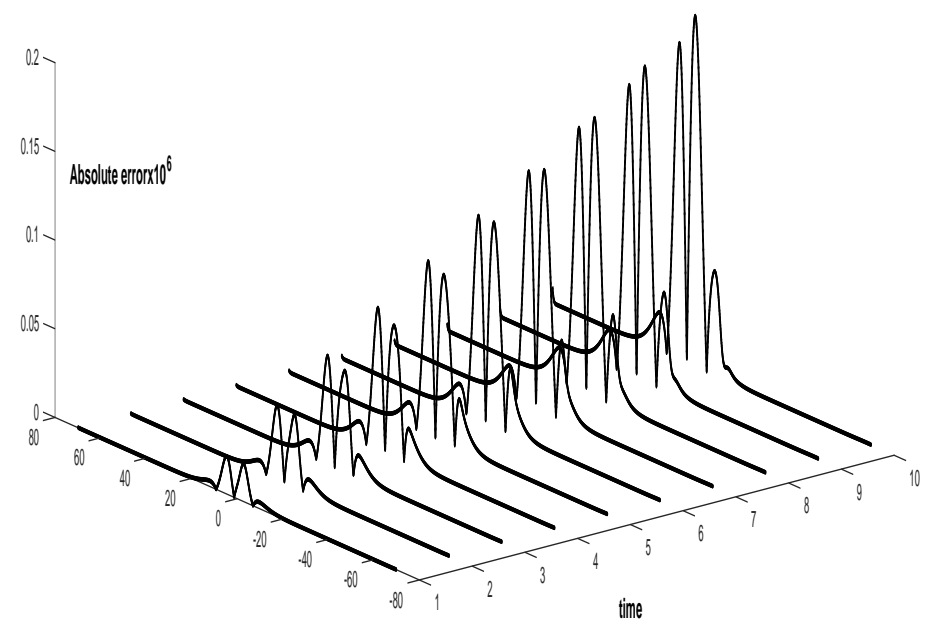

Figure 2. The graphics of absolute error for $h=0.05$ and $\Delta t=0.001$ at $t_{\text {final }}=10$.

From the obtained results, it is clearly seen that the presented method produces better results than those of previous studies. The advantage of the present method is obviously seen when the error norms $L_{2}$ and $L_{\infty}$ are compared with those of other studies. The low computational cost and storage requirement of the method can be stated as its most important advantages. The schemes of the present method can easily be applied by using modern symbolic programming languages. 
Table 6. The peak points of the wave for $h=0.01$ and $\Delta t=0.001$.

\begin{tabular}{lll}
\hline $\mathrm{x}$ & Time & Peak point of wave \\
\hline \hline 0.0 & 0 & -0.84526249034 \\
1.10 & 1 & -0.84524510909 \\
2.30 & 2 & -0.84521217373 \\
3.40 & 3 & -0.84525394060 \\
4.50 & 4 & -0.84526094228 \\
5.60 & 5 & -0.84523317811 \\
6.80 & 6 & -0.84522827511 \\
7.90 & 7 & -0.84525965995 \\
9.0 & 8 & -0.84525627990 \\
10.10 & 9 & -0.84521813561 \\
11.3 & 10 & -0.84524126975 \\
\hline
\end{tabular}

\section{Conclusion}

In the present study, two reliable methods, namely finite element quintic B-spline collocation and Strang splitting method are combined in order to find the numerical solutions of the modified Fornberg-Whitham Equation. Firstly, the considered equation is transformed into its linear and nonlinear parts as subproblems, then the spatial domain is divided into $N$ equal elements to apply the collocation method. The application of the methods is carried out successfully to obtain accurate numerical results. The stability of the numerical scheme is analyzed via Von Neumann method. Additionally, graphical simulations of new numerical results and error norms are presented. A comparison of the present results with those of some previously published ones shows that the presented method has produced acceptable results. The calculated error norms $L_{2}$ and $L_{\infty}$ also show the accuracy and efficiency of the present study. The figures and tables show the newly obtained results at various times and show the consistency of the newly results. All the results in this study found out that finite element collocation method with Strang splitting technique has many advantages over other studies due to its effectiveness, applicability and accuracy. Thus, the presented method can be applied to a wide range of problems arising in science.

\section{References}

[1] Abidi, F., Omrani, K.: The Homotopy Analysis Method for Solving the Fornberg-Whitham Equation and Comparison With Adomian's Decomposition Method. Comput. Math. Appl. 59 (8), 2743-2750 (2010).

[2] Bariza, B., Akgul A., Inc M.: New approach for the Fornberg “Whitham type equations. J. Comput. Appl. Math., 312 (1), 13-26 (2017).

[3] Jafar, B., Eslami, M.: Approximate solutions for Fornberg-Whitham type equations. Internat. J. Numer. Methods Heat Fluid Flow, 22 (6), 803-812 (2012).

[4] Nuseir, A.S.: New exact solutions to the modified Fornberg-Whitham equation. Taiwanese J. Math., 16 (6), 2083-2091 (2012).

[5] Chen, A., Li, J., Deng, X., Huang, W.: Travelling Wave Solutions of the Fornberg-Whitham Equation. Appl. Math. Comput. 215 (8), 3068-3075 (2009).

[6] Fornberg, B., Whitham, G.B.: A numerical and theoretical study of certain nonlinear wave phenomena. Philos. A Trans. R. Soc. London (1978).

[7] Gupta, P.K.,Singh, M.: Homotopy Perturbation Method for Fractional Fornberg-Whitham Equation. Comput. Math. Appl. 61 (2), 250-254 (2011).

[8] Geiser, J.: Decomposition methods for differential equations: theory and applications. CRC Press. London (2009). 
[9] He, B., Meng, Q., Li, S.: Explicit peakon and solitary wave solutions for the modified Fornberg-Whitham equation. Appl. Math. Comput. 217 (1), 1976-1982 (2010).

[10] Hesam, S., Nazemi, A., Haghbin, A.: Reduced Differential Transform Method for Solving the Fornberg-Whitham Type Equation. Int. J. Nonlinear Sci. 13 (2), 158-162 (2012).

[11] Lu, J.: An analytical approach to the Fornberg Whitham type equations by using the variational iteration method. Comput. Math. Appl., 61 (8), 2010-2013 (2011).

[12] Marchuk, G.I.: Some application of splitting-up methods to the solution of mathematical physics problems. Aplikace Matematiky. 13 (1), 103-132 (1968).

[13] Prenter, P.M.: Splines and Variational Methods. Wiley Publications. New York (1975).

[14] Ray, S., Gupta, A.K.: Numerical Investigation of Time- Fractional Modified Fornberg-Whitham Equation for Analysing the Behaviour of Water Waves. Appl. Math. Comput. 266 (1), 135-148 (2015).

[15] Strang, G.: On the construction and comparison of difference schemes. SIAM J. Numer. Anal. 5 (3), 506-517 (1968).

[16] Zhou, J., Tian, L.: Type of Bounded Traveling Wave Solutions for the Fornberg-Whitham Equation. J. Math. Anal. Appl. 346 (1), 255-261 (2008).

\section{Affiliations}

NURI MURAT YAĞMURLU

ADDRESS: İnönü University, Dept. of Mathematics, 44280, Malatya-TURKEY.

E-MAIL: murat.yagmurlu@inonu.edu.tr

ORCID ID: 0000-0003-1593-0254

ERSIN YILDIZ

ADDRESS: İnönü University, Dept. of Mathematics, 44280, Malatya-TURKEY.

E-MAIL: 36173614027@ogr.inonu.edu.tr

ORCID ID: 0000-0003-4748-4738

YUSUF UÇAR

ADDRESS: İnönü University, Dept. of Mathematics, 44280, Malatya-TURKEY.

E-MAIL: yusuf.ucar@inonu.edu.tr

ORCID ID: 0000-0003-1469-5002

AlaAtTin ESEN

ADDRESS: İnönü University, Dept. of Mathematics, 44280, Malatya-TURKEY.

E-MAIL: alaattin.esen@inonu.edu.tr

ORCID ID: 0000-0002-7927-5941 